\title{
IMPORTANCE OF DRONES IN HEALTHCARE DELIVERY AMID A PANDEMIC: CURRENT AND GENERATION NEXT APPLICATION
}

\author{
${ }^{1}$ Department of Medical Laboratory Science, University of Benin, Nigeria. \\ Corresponding Author Email: oigbochieanita@yahoo.com Phone: +2348084447975
}

${ }^{1}$ Odigie, E. B., ${ }^{1}$ Adejumo, B. I. G., \& ${ }^{1 *}$ Oigbochie, A. E.

\begin{abstract}
A drone is an aircraft controlled from a distance without a human pilot onboard using devices ingrained with software flight protocols. Outside the military, it lately just started to reach the relevancy of conventional businesses. The healthcare sector is an industry where this capacity is needed particularly in a pandemic in providing a responsive healthcare service in a contact-free manner. Also, delivery of blood supplies, vaccines, medications and other medical supplies to rural areas with no access to health facilities is made possible by the use of drones as they can navigate through difficult barriers such as buildings and bridges as well as areas that have been rendered inaccessible by conventional land and air transport. Drones have triggered a swift advancement in a variety of commercial, recreational and industrial applications with its capability to effectively put together real-time information on events at a low cost. However, its breakthrough in healthcare has been slower compared to other sectors where drones have been used extensively. Hence, this review is aimed at emphasizing the importance of drones in healthcare delivery noting its current and future usability. In this paper, a systematic review of relevant literatures was employed. Findings suggests that drones have been used effectively in healthcare viz a viz: enabling transportation of birth control, biopsy for emergency surgeries and other medical and laboratory supplies within few minutes. Considering the current global situation (COVID-19 pandemic), the future might experience more outpatient care and perhaps home-based care previously admitted in hospitals. Test results, medications and other treatment ordered by physicians may be couriered to patient's residence via drones. In conclusion, drones offer a variety of exciting opportunities; delivery of medical and laboratory supplies, blood products including biopsy for emergency surgeries is just a starting point. Drones have the capability to evolve medical care as well as propel advancement in the health industry.
\end{abstract}

Keywords: Drones, Healthcare delivery, Medical importance, Pandemic

LICENSE: This work by Open Journals Nigeria is licensed and published under the Creative Commons Attribution License 4.0 International License, which permits unrestricted use, distribution, and reproduction in any medium, provided this article is duly cited.

COPYRIGHT: The Author(s) completely retain the copyright of this published article.

OPEN ACCESS: The Author(s) approves that this article remains permanently online in the open access (OA) mode.

QA: This Article is published in line with "COPE (Committee on Publication Ethics) and PIE (Publication Integrity \& Ethics)". 


\section{INTRODUCTION}

Drones are conventionally referred to as UAVs and UAS which means unmanned aerial vehicles and unmanned aircraft systems respectively. Just like unmanned aerial vehicles and unmanned aircraft systems, other names used in place of the term drone are: RPV and RPAS, meaning remotely piloted vehicle and remotely piloted aircraft system respectively. Remotely piloted aircraft system is a more multinational and conventional term while remotely piloted vehicle is most often associated with military environments (ACUO, 2014). Basically, a drone is a form of robot designed to be flown by controlling it from a distance using a computer or devices with software-controlled flight plans programmed in them, operating simultaneously with built-in sensors and global positioning system (GPS). This however is done without a human pilot on board, hence the name unmanned aircraft (FAS, 2017).

Historically, unmanned aerial vehicles were usually connected with the military, where before now, were used more contentiously as a platform for weapons as well as in military intelligence for gathering information about a particular entity and for anti-aircraft target. In recent time, drones have been employed in an extensive range of activities outside military roles with the line up from surveillance, traffic and weather monitoring, search and rescue, firefighting, photography/videography, agriculture, delivery services as well for personal and other commercial purposes (Rouse et al. 2019). This unmanned aircraft (drone) is basically made up of a propulsion system, an air frame and navigation system which consist of an extensive range of supporting equipment and aircraft layout settings that facilitates several application programs. It lately just begun to reach the relevancy of conventional businesses despite not being a new technology by delivering a cost effective, swifter and preferable choice compared to the manned aircraft (FAS, 2017).

As at now, a disease caused by the virus known as coronavirus disease 19 or simply COVID-19 is currently ravaging the world at an alarming pace and as a result of the devastating prowess of the causative organism (SARSCoV-2); many hospitals are reported to have broken down due to the overwhelming influx of affected persons (Wong et al. 2020). As of the time of writing this article, SARS-CoV-2 has infected more than 25 million of the world's population and close to one million people have died of COVID-19 in approximately 216 countries (WHO, 2020). In the face of COVID-19, there have been little or no reports as far as we know, on using drones to combat or support healthcare workers who are continually being exposed to COVID-19 without remorse. Drones have triggered a swift advancement in a variety of commercial, recreational and industrial services with the capability to put together real time data cost effectively as well as transport air cargo. However, its breakthrough in healthcare has been slower compared to other sectors where drones have been used extensively. Overall, drones have the capability to evolve medical care and propel advancement in the health industry.

It is against this backdrop that we intend to create awareness on the importance of drones in healthcare delivery particularly in the era of COVID-19 while looking at its current and future applications.

\section{TYPES OF DRONES \\ MULTI ROTOR DRONES}

Amongst other types of drones, the multi rotor drone is the most predominantly used by amateurs, professionals, hobbyists and drone enthusiasts with similar interest. They are mostly utilized in conventional activities such as 
aeronautic videography and photography as well as in surveillance. Albeit somewhat cheap and simple to build, multi rotor drone has a number of drawbacks. The well-known downsides are its circumscribed speed, flying time and endurance making it unsuitable for wide-reaching projects that requires long haul flying (Figure 1A). The major problem associated with the multicopters is that they spend a great amount of their energy trying to balance themselves in the air and oppose gravitation. This however probably exhausts the battery that would have been channeled into their flying time. Notwithstanding, their ability to remain still in air makes them useful in aerial photography and other applications that require hovering in the air for a long period of time. As of now, a good number of the multi rotor drones available have a flying time of about 20 to 30 minutes and can mostly afford to fly a light weighted air cargo as in a camera (CircuitsToday, 2018).

\section{FIXED WING DRONES}

Unlike the multi-rotor type of drones, fixed wing drones are built differently and also differ completely in terms of design. Just like normal manned aircrafts, they have wings and with regards to flying and air control, fixed wing drones do not exhaust their energy trying to remain balanced in the air opposing gravitation unlike the multi-rotor drones. Fixed wing drones cannot remain still on the air rather they fly progressively under the guidance of a remote controller within the set time the energy life allows (Figure 1B). Owing to their inability to stand still in air, they cannot be utilized in applications that require remaining suspended in air for a long period such as in aerial photography. However, a good number of fixed wing drone models have a higher-flying time and are ideal for applications that require long haul flight such as in aerial surveillance and mapping. It has a typical flying time of a couple of hours and utilizes energy (fuel/battery) efficiently and effectively (CircuitsToday, 2018).

\section{SINGLE ROTOR DRONES}

Single rotor drones like the name implies, have only one big sized rotor and a small sized rotor at the extremity of the drone to help navigate its direction. Unlike the multi rotor models, they are more efficient and productive with a higher-flying time and can be energized by gas engines (Figure 1C). Their appearance, structure and built closely resembles that of a real-life helicopter. The complexities and operational risks associated with these single rotor drones are however more advanced. They are also more expensive and require professional training to fly them appropriately in air although a runway or a catapult launcher may not be required to launch them on air (CircuitsToday, 2018).

\section{HYBRID VTOL}

These hybrid models, combines the advantages of both the rotor-based versions and the fixed wing versions. The rotor models in terms of their ability to remain still in air while the fixed wing models for their higher-flying time. They are characterized of both manual gliding and automation. For the manual, it utilizes a remote based manual control which may also be programmed to navigate the drone on a set course or direction. Whilst the automation consists of the gyros and accelerometers that utilizes the autopilot concept (automated mode) to ensure the drone stability in air (Figure 1D). There are a number of this hybrid VTOL's obtainable for commercial use with the Amazon commercials being the most common for its top-tier delivery services (CircuitsToday, 2018). 

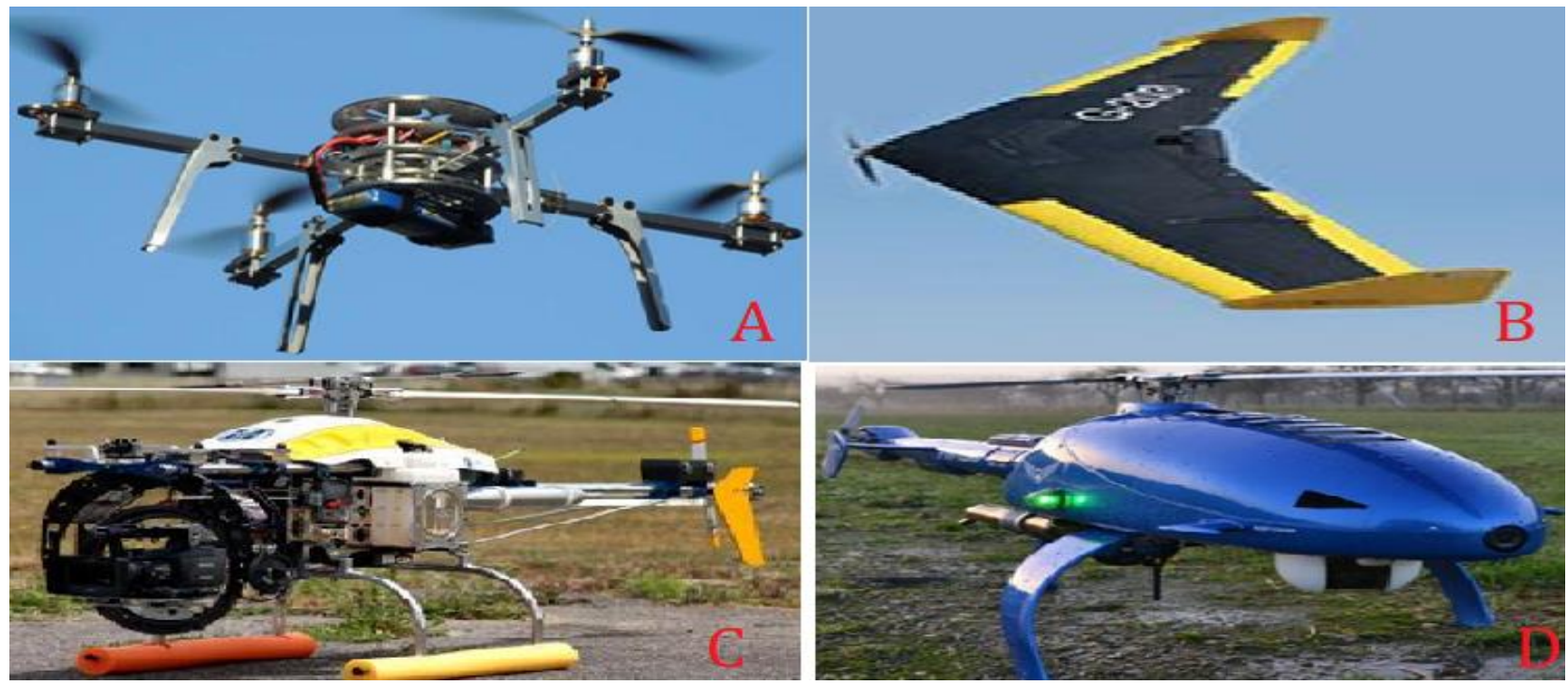

Figure 1: Types of drones currently in use: A (Multi Rotor), B (Fixed Wing), C (Single Rotor) and D (Hybrid VTOL). Source: CircuitsToday, 2018.

\section{DATA SOURCES}

In this paper, a systematic review of existing research papers was used, relevant data from scholarly literatures were retrieved and analyzed. After a thorough review, detailed information relevant to this paper was retrieved from all sourced papers upon which this paper is developed.

\section{OBSERVATION}

Findings from the review of sourced data suggests that drones have been used in some places effectively in healthcare delivery where it successfully enabled transportation of blood products, vaccines, medications, birth control and other medical and laboratory supplies across distances in a short time.

\section{DISCUSSION \\ DRONE'S BENEFITS}

Adverse weather or poor road and air conditions such as icy patches, rain, snow, dust, fog and ice puts delivery vehicles and aircrafts at risk of danger and hazards as well as render delivery services unworkable. Traffic situations inflicts terrible delays in delivery services. Drones however proffer efficacious and efficient replacements in these conditions and are presently utilized on the front line of disasters. Drones can only fly a maximum of 400 feet above the ground making it easier to maneuver fogy skies unlike with helicopters or airplanes (Graboyes and Bryan, 2019; Hamilton, 2020). Using helicopters and airplanes for delivery is very time consuming as they have to fly in from other locations and then do their work. Drone have been used efficiently to carryout missions that were originally completed by helicopters and airplanes which are more expensive to hire (Corrigan, 2019). Drones are cost effective and budget friendly for example, a 3-ton helicopter or 2-ton vehicle are more expensive for delivery services compared to a 25 pound drone and also the cost of drones are significantly less expensive than vehicles and aircrafts. Also, drones do 
not require humans onboard hence there will be no need employing someone for the job cutting down expenses. In terms of delivery time, vehicles would take longer time to get to delivery destination, and considering bad roads and traffic conditions, delivering on time is almost impossible. Penetrating or maneuvering constricted spaces is achievable owing to drone small sizes. With other conventional means of transport, there are some safety risks involved for instance, crew members and passengers in helicopters or airplanes are at risk of air crash whilst in the air. Just as with these aircrafts, drivers as well as passengers are also at risk of accidents or any form of car crash whilst delivering supplies. Drones are effective in mitigating these aforementioned safety issues. With drones, pilots can stay safely on the ground while navigating its direction thereby eliminating risk to human passengers (Brown, 2015; Graboyes and Bryan, 2019).

\section{DRONE'S APPLICATION IN HEALTHCARE DELIVERY}

We are in a digital age where technology is improving; a drone is one aspect of this technology that is yet to be embraced in the health sector. Drone technology can help the health industry improve care delivery and safety with regards to rapid delivery of vaccines, medications and other health supplies to areas where they are needed in time preventing an outburst of deadly communicable illnesses. Medical professionals ranging from paramedics to other health workers in primary health centers to large hospitals are faced with loads of challenges every day, which may include the ability the reach patients in dare need of immediate medical attention. The distance between these patients and the health professionals could mean the difference between survival and death. Drones can help provide efficient health care to patients within a short period of time. Delivery of blood supplies, vaccines, medications, snake bite serum and other medical supplies to villages and rural areas with no access to health facilities may only be made possible with a drone.

Drones can fly in many places that manned aircrafts can't, with its ability to navigate through difficult barriers like buildings and bridges as well as delivering these supplies to areas with no accessible roads. Within hospital walls, drones can help transport medications to patient's bed side as well as courier blood for transfusion between hospitals in a timely fashion. Unmanned aerial vehicles offers a wide range of possibilities to the healthcare industry, possibilities that are cost effective as well as life-saving. Medications, communication equipment, portable shelter, mobile technology and other essentials comprise the long list of items that could be transported via a drone without delay to areas that have been rendered inaccessible by conventional land and air transport as a result of disasters, pandemics or other tragedies. The ability to travel a long distance effectively in a short time to deliver laboratory samples, blood products, medications and other essentials to areas needed is a great advantage. Unmanned aircraft systems offer these opportunities, which is why researchers, manufacturers and other organizations are beginning to delve into the drone technology to provide applications that enhance productivity and improve medical outcomes (Tucker, 2017).

\section{TRANSPORTING BLOOD AND RELATED SUPPLIES}

With the integration of drones into the health care industry, the first area that would experience the bulk of its benefits is delivery. Countries that have experienced a bit of the benefit of these unmanned aircraft applications are beginning 
to carry out extensive research in this regard (Tucker, 2017). Stan Brock, founder and president of Remote Area Medical (RAM), a nonprofit organization that provides medical care to remote areas who begun an air ambulance operation, a service that delivers vaccines and medications to remote villages using the manned fixed-wing helicopters- flying every two days at a costly rate, first became interested in the drone application for delivering supplies just after he began the air ambulance operation. In his words, it would be extremely helpful if we were able to send packages of medicine and vaccines over great distances by drone. In that part of Virginia (where Let's Fly Wisely took place), a great population inadequately provided with services cannot get out of the house during inclement weather, but are in desperate need of blood pressure medicine or other medications that may be required urgently, a drone could deliver those medications to these areas where vehicles wouldn't be able to access as these unmanned aircrafts can maneuver adverse weather conditions that would delay multiple deliveries using manned aircrafts which is more costly and complex in operation. Strege reckoned that the drone application is perfect for the initiative Remote Area Medical (RAM) recently embarked on in Philippines where they will require to deliver medical supplies across a wide stretch or length of water.

In an encouraging phenomenon, Dr. Mark Head, foreign crisis coordinator, Greece, made a remark on equipping the unmanned aircrafts with defibrillators alongside the medical supplies. He reported that this will be a progressive step in saving lives and maximize time, which is the primary aim of any health professional in an emergency situation. Alas, work on manufacturing the first unmanned aerial system (UAS) prototype with a defibrillator began and was carried out by Alec Mormot, a graduate industrial design student at TU Delft University in Holland. A smart phone application can be used to call the drone in case of an emergency in his prototype. On arrival, guidance or steps on how to use the machine can be given to whomever is present at the emergency scene by medical personnel through the phone. According to Dr. Mark, getting a drone equipped with a defibrillator to an emergency scene with a person having a cardiac arrest faster than an ambulance can save lives. Getting the defibrillator to the emergency scene quickly is a step towards survival, it is the time after the event that is critical (Knight, 2016). In a particular scenario, which occurred in July 2019, where in a medical doctor suffered a cardiac arrest, collapsed and eventually died in a Federal Medical Centre, Yenogoa Nigeria, due to lack of a defibrillator. In a swift reaction, medical team on ground had to travel a long distance to get to the nearest hospital in possession of the equipment (Government House Clinic) but it was rather too late as the doctor had died before the team returned. This simply means that the presence of a drone to transport the defibrillator could have saved the doctor's life. This is one in thousands of cases that occur yearly in different parts of the world but particularly in developed countries.

\section{HOSPITAL DELIVERIES}

At present, a good number of researches associated with healthcare related unmanned vehicles are inclined towards delivery of medical supplies to rural areas with no health care facility, with less attention to other areas in the healthcare industry that can benefit from this drone application (Graboyes and Bryan, 2019). The founder of the consulting company Wilstair, Will Stavanja, is amongst the few researching on other ways drones can be useful in the hospital. According to Stavanja, amongst those ways is by transporting medications and blood samples from floor to floor or between buildings rather than the conventional way which involves transporting by foot or by pneumatic tube 
transportation as done in some hospitals (Knight, 2016). Employing drones in delivery of specimens and medications within hospital walls and buildings is more budget friendly compared to expanding a pneumatic tube system. Even though Stavanja is excited about the potential usability of this application inside the hospital, the limitations are also a cause for concern. For instance, communication system: outdoor drones depend on radio frequencies and global positioning system (GPS) signals to accomplish their tasks, both of which have restrictions indoors (Knight, 2016). A solution to that limitation might be the Bluetooth technology, however, researchers need to find a way to work through fully automating drones so they are more aware of their environment and know the location they are in. There is also the challenge with weight. The smarter the drone, the bigger it is and the more costly also, but to cut cost, these UAS will have to be small (Graboyes and Bryan, 2019).

Another challenge is that staffs are unwilling to adopt this technology because they are concerned that it would put their jobs on the line and perhaps replace them. Stavanja argued that it will only avail them the time to focus better on other tasks (Hlad, 2015). Also, to ensure the drones are transporting the appropriate package at the appropriate temperature to the required location, it will be necessary for people to create flight path and manage the drones. Drones can take on some of the smaller roles such as transporting medications from floor to floor. Medical personnels can request for certain items to be delivered to a particular ward or room via a smart phone application and the drone will be programmed for the task. This however avails the hospital the privilege to indulge existing employees in other tasks as well as meet other hospital demands (Knight, 2016). Personal protective equipment as well as other medical supplies are being delivered to a medical facility (Novant Health Medical center) in charlotte, North Carolina via Zipline's drones. The deliveries are wholly done in a contact free manner, especially considering the COVID-19 pandemic. According to zipline, this is the first emergency drone logistics operation to assist health centers in tackling the pandemic and it is also the longest-range unmanned aircraft delivery service in the US that has been approved. Additionally, in a statement by Keller Rinaudo, Zipline's CEO, he emphasized on the importance of using contact free drones in tackling the COVID-19 pandemic as we are likely in for a long-term fight against it. However, the work in progress in Novant Health center in North Carolina will serve as a model to the rest of the country in terms of putting together the most responsive and resilient health care system possible (Porter, 2020).

\section{SURVEILLANCE OF DISASTER SITES}

In surveillance of disaster sites drones are useful in tracking disease outbreaks as well as areas with chemical and biological hazards. From research, drones have been effective in gathering real time information involving data of victims and patients in high-risk environments in need of urgent medical attention (Hlad, 2015). The National Health Service in England has looked into the efficacy of drones in evaluating injuries related to biological, chemical and nuclear materials with the purpose of improving the orderliness and effectiveness of emergency response teams (Aron, 2016). The UAS technology has been effective in detecting materials that are hazardous to health such as aerosols, heavy metals and radiation. Drones equipped with high-resolution photogrammetry operating system were employed to effectively evaluate and predict the risk of developing cancer in agricultural environments with elevated copper concentration in a study carried out in Southern Italy (Capolupo et al., 2015). An experiment was carried out to demonstrate the efficacy of a multi-rotor drone with an in-built sampling platform to effectively measure trace gas 
levels and aerosol within complex geographic areas. Employing this system can prevent the outburst of health hazards from pathogens if detected in time (Brady et al., 2015).

Amidst all these, drone technology has also been used in mapping out radiation from uranium mines as well as in detecting radionuclides that are typical in nuclear accidents (Martin et al., 2015). In addition, drone application has proven usable for epidemiology study based off of its ability to gather high-resolution, real-time and space-based information at an inexpensive rate. This is applicable in agricultural expansion, monitoring deforestation as well as other practices that make changes to the ecosystem and environmental area. There was also a demonstration in Malaysia on the use of drones in characterizing deforestation patterns and changing land that influences the transmission of diseases from animals or insects to humans such as in malaria parasite (Fornace et al., 2014). In recent time, drones with nucleic acid analysis modules have been used by researchers in detecting Ebola virus and Staphylococcus aureus (Priye et al., 2016).

\section{TELEMEDICINE}

Telemedicine is a procedure that allows health professionals to evaluate, diagnose and proffer treatment to patients in remote areas especially, using telecommunications technology. This field of telemedicine can be made more auspicious by incorporating the drone technology into it (Breen et al., 2010). Telemedicine allows patients to access medical expertise efficiently and swiftly without having to travel. Sadly, communications required to accomplish this purpose to remote, rural district, disaster-relief, havoc or war zone areas cannot be subject to commercial networks. In tele mentoring, an experienced health personnel or proceduralist, provides guidance with emerging procedures to a colleague with less experience by means of telecommunications technology and computers (Rosser et al., 1997).

Applying this information technology concept, the use of drones in establishing a wireless networking to carry out telesurgery between a surgeon and a robot was demonstrated. Telesurgery involves performing surgical procedure using robotic technology with the surgeon and patient distantly located from each other (Harnett et al., 2008). Less extreme case scenarios have been looked into in recent time with telemedical drones tested by William Carey University College of Osteopathic Medicine to deliver medical supplies and communication packages for urgent clinical scenarios, helping in providing care (AOA, 2016).

\section{APPLYING DRONES FOR MEDICAL LABORATORY SERVICES}

Accessing laboratories and specimen delivery for laboratory analysis from research, were amongst the biggest issues faced by people worldwide as a result of bad infrastructure, traffic and inaccessibility. This is applicable to both rural and urban areas particularly in Nigeria, because surprisingly the so-called big cities have terrible roads and are faced with traffic daily. There is a need for drones, to enable access to health care services in these places especially in remote and rural districts. Medical laboratory is a key aspect in health care with regards to test results. Some medical centers are primarily collection sites, a large portion of specimens are collected in one location and delivered to a different location for analysis and diagnosis. Some laboratory investigations are carried out exclusively in specific centres and as such, requires transporting such specimens over a long range. Speed is of importance, if specimens stay unattended to for a long while, eventually the specimen starts deteriorating and it becomes not so relevant for analysis. 
Also, sending test results to the physician in a timely fashion is very essential in-patient care for diagnosis and treatment of disease. There are also cases of emergency where transfusion needs to be done but no matching blood in the blood bank of the facility, a drone can be used to deliver blood from neighboring blood banks within a short time. That said, Medical Laboratory is another important area in healthcare industry where this capacity is needed. Also, in Histopathology, samples for histology can take days or even weeks before results are issued. However, there are cases of urgency where histology results are required for immediate diagnosis and treatment. Take for example a biopsy required to be moved for urgent histological examination. An absence of obstruction would hasten the process. This also, is another area where medical drones are relevant. A drone is a reliable transportation service that can be utilized for a wide range of purposes.

\section{MAJOR CHALLENGES}

Implementing the unmanned aerial system comes with some limitations, and this is applicable to every industry interested in this technology. Tucker (2017), noted that these challenges may range from battery life to payload capacity as well as its regulations. These regulations place constraints in rounding up testing on health-related delivery services of drones in the U.S although a number of researches carried out in this regard is being done in other countries. Albeit changes have been made on the Part 107 regulation by the Federal Aviation administration (FAA), with the release of a new Part 107 regulation removing some limitations, there are still uncertainties. He also envisages more interest in this technology in the future by different industries owing to these limitations being worked on appropriately, with the industries enjoying the benefits associated with this application from delivery services to more advanced services. The biggest hindrance to the drone technology is the federal aviation administration, however there have been improvements (Tucker, 2017).

There are worries on privacy invasion, as the drone technology may encourage remote spying by unknown entities. There are also fears that the airspace will be littered with aircrafts impeding movements as well causing collision between manned and unmanned aircrafts. Other concerns include drones striking buildings and flying over or falling on people. Recommendations on the effective operation of the drone technology was suggested by the former U.S. special operations Command director for unmanned aerial systems. He is of the opinion that ground-to-drone communications should be secured to avoid hackers from taking control of the unmanned aircrafts and also prevent cybercrimes. Also flying drones beyond the operator's line of sight will make it more complicated as well as increase pilot-to-drone cost. Thirdly, drones will require a kind of wireless network to incorporate them into the national air control system. Finally, drones manufactured for medical use should be able to carry out expeditions more efficiently than what the United State presently anticipates from conventional amateur drones (Robert and Darcy, 2019).

\section{THE FUTURE}

There are optimistic expectations for the drone technology and also future predictions are aggressive with the drone market envisaging small indoor drones capable of delivering medications from the pharmacy to the patient's bedside as well as to them in the comfort of their homes providing home based health-care services rather than the typical hospital-based service. The future will experience more outpatient care and perhaps home-based care previously done 
in the hospital. Providing home-based care securely without risk and effortlessly for a number of circumstances can be achieved using drones. After home consultation and clerking by a physician on a patient, samples can be taken from the patient and delivered at once via a drone to the laboratory for analysis. After test results have been issued out, medications and other treatment ordered by the physician can also be transported back to the residence of the patient via a drone.

Also, people in assisted living facilities will now get home-based care for a prolonged time enabling them depend less on others for support, credits to this technology. Drones can be used to deliver food to people who are ill and cannot prepare their meals as well as keep watch on home patients with medical conditions that interfere with their daily functioning. With the growth of this technology, a lot of prospective clients have gone pass the inspection and background check phase with a number of industries coming to terms with the reality of drone efficiency and benefits as they turn to this technology for secure and budget friendly missions. This however will help propel swift revolution and encourage advancement in the industry (Tucker, 2017). Also, with restriction in movement and lack of access to health care facilities during pandemics as in COVID-19, drones will be a very effective means of delivery as well as provide home-based health care in a contact free manner.

\section{CONCLUSION AND RECOMMENDATIONS}

Drone usability is on the rise as it offers a variety of exciting opportunities. Delivery of medical and laboratory supplies, blood products, including biopsy for emergency surgeries is just the starting point. Drones utilized for medical supplies are the aid and future of remote areas that have been rendered inaccessible by conventional land and air transport as a result of disasters, havoc, bad infrastructure, war and other tragedies and also a relief for people faced with inclement weather and pandemics- in desperate need of services but are stuck with strict lockdown policies. In a number of places worldwide, health professionals such as medical laboratory scientists, physicians, nurses and other medical personnel are starting to experience drone utility in rendering services. The exploitability of drone technology is unascertained and extensive.

Drones have the capability to evolve medical care as well as propel advancement to the health industry. Drones have the capacity to be an authentic mode of delivery in the medical industry for Laboratory samples, pharmaceuticals, vaccines, emergency medical equipment, and ambulance services. Government agencies worldwide should place drone use on a global agenda especially considering the present reality. With restriction in movement and lack of access to health care facilities during pandemics as in COVID-19, drones will be a very effective means of delivery as well as provide home-based health care. There should be accelerated research initiatives in the areas of safety and airspace management to ensure a workable traffic management system. For privacy concerns, strict rules on registration and licensing of drones at an affordable rate should be encouraged to enable close monitoring, tracking and identification in case of suspected crimes and intrusion. There should also be increased public awareness and manufacturers should look into manufacturing drones that can accommodate bigger payload and of course drones with better battery life. 


\section{AUTHORS' CONTRIBUTIONS}

This paper is in full collaboration among authors and we accept any responsibility in full. All authors contributed immensely to justify authorship. AEO and EBO conceptualized the review article while all authors defined the intellectual content. AEO, BIGA and EBO managed the literature searches; all authors drafted the manuscript and contributed to the overall analysis. All authors revised, reviewed and approved the final draft. AEO handles the correspondence on behalf of other authors.

\section{REFERENCES}

American Osteopathic Association (AOA), (2016). Osteopathic Emergency Physician Launches New Medical Disaster Drone for audience of Homeland Security, Global Health Organisations. Retrieved on $28^{\text {th }}$ January, 2021 from: https://www.prnewswire.com/news-releases/osteopathic-emergency-physician-launches-newmedical-disaster-drone-for-audience-of-homeland-security- global-health-organizations-300373985.html/

Aron, J. (2016). NHS to use drones to help chemical, bio and nuke response teams. New scientist. Retrieved on $21^{\text {st }}$ January, 2021 from: https://www.newscientist.com/article/2075625-nhs-to-use-drones-to-help-chemicabioand-nuke-response-teams/

Australian Certified UAV Operators Inc. (ACUO), (2014). UAV, UAS OR RPAS. Retrieved on 22nd January, 2021 from:

https://www.infrastructure.gov.au/aviation/asrr/public_comments/files/Australian_Certified_UAV_Operators _Inc.pdf

Brady, J., Stokes, D., Bonnardel, J. and Bertram, T. (2016). Characterization of a quadrotor unmanned aircraft system for aerosol-particle-concentration measurements. Environmental Science Technology. 50:1376-1383.

Breen, G.M. and Matusitz, J. (2016). An evolutionary examination of telemedicine: a health and computer-mediated communication perspective. Social Work Public Health. 25:59-71.

Brown, J. (2015). BENEFITS OF DRONE TECHNOLOGY. Retrieved on $5^{\text {th }}$ January, 2021 from: https://www.mydronelab.com/blog/drone-uses.html

Capolupo, A., Pindozzi, S., \& Okello, C. (2015). Photogrammetry for environmental monitoring: the use of drones and hydrological models for detection of soil contaminated by copper. Science Total Environment. 514:298306.

CircuitsToday (CT), (2018). Types of drones- Explore the Different Models of UAV's. Retrieved on $21^{\text {st }}$ January, 2021 from: htpp://www.circuitstoday.com/types-of-drones

Corrigan, F. (2019). What are Drones Used from Business to Critical Mission. Retrieved on $20^{\text {th }}$ January, 2021 from: https://www.dronezon.com/drones-for-good/what-are-drones-used-for-and-best-droneuses/

Federal Aviation Administration (FAA), (2019). Unmanned Aircraft Systems (UAS). Retrieved on $1^{\text {st }}$ February, 2021 from: https://www.faa.gov/uas/

Federation of American Scientists (FAS), (2017). Unmanned Aircraft System (UAS) Service Demand 2015-2035 
Literature Review and Projections of Future Usage. Washington DC: Retrieved on $18^{\text {th }}$ January, 2021 from: https://fas.org/irp/program/collect/service.pdf

Fornace, K., Drakeley, C., William, T., Espino, F. and Cox, J. (2014). Mapping infectious disease landscapes: unmanned aerial vehicles and epidemiology. Trend's parasitology. 30:514-519.

Graboyes, R. and Bryan, D.N. (2019). Drones delivering medical supplies and more can help save American lives. Retrieved on $4^{\text {th }}$ February, 2021 from: https://www.statnews.com/2019/01/18/drones-delivermedical-supplies-united-states/

Hamilton, J. (2020). Public Benefits of Drone Technology. Retrieved on 6th January, 2021 from https://www.roboticstomorrow.com/article/2020/03/public benefits-of-drone- technology/15025

Harnett, B., Doarn, C., Rosen, J., Hannaford, B. and Broderick, T. (2008). Evaluation of Unmanned airborne vehicles and mobile robotic telesurgery in an extreme environment. Telemedicine Journal of Environmental Health. 14:539-544.

Hlad, J. (2015). Drones: A Force for Good When Flying in the Face of disaster. The Guardian. Retrieved on $14^{\text {th }}$ January, 2021 from: https://www.theguardian.com/global-development/2015/jul/28/drones-flying-in-theface-of-disaster-humanitarian-response/

Knight, R. (2016). Drones Deliver Healthcare. Retrieved on $2^{\text {nd }}$ February, 2021 from: https://insideunmannedsystems.com/drones-deliver-healthcare/

Martin, P.G., Payton, O., Fardoulis, J. and Scott, T. (2015). The use of unmanned aerial systems for the mapping of legacy uranium mines. Journal of Environmental Radioactivity. 143:135-140.

Porter, J. (2020). Zipline's drones are delivering medical supplies and PPE in North Carolina.

Retrieved on $6^{\text {th }}$ January, 2021 from https://www.google.com/amp/s/www.theverge.com/platform/amp/2020/5/27/21270351/zipline-dronesnovant-health-medical-center-hospital-supplies-ppe

Priye, A., Wong, S. and Bi, Y. (2016). Lab-on-a-drone: toward pinpoint deployment of smartphone-enabled nucleic acid-based diagnostics for mobile health care. Analytical Chemistry. 88:4651-4660.

Rosser, J., Wood, M. and Payne, J.H. (1997). Telementoring: a practical option in surgical training. Surgical Endoscopy. 11:852-855.

Rouse, M., Dennis, S., Alan, E., Shea, S. and Wigmore, I. (2019). Drone (UAV). Retrieved on $28^{\text {th }}$ January, 2021 from: https://internetofthingsagenda.techtarget.com/definition/drone

Tucker, J. (2017). Drones in Healthcare. Retrieved on $13^{\text {th }}$ February, 2021 from: https://www.dronesinhealthcare.com

Wong, S.C.Y., Kwong, R.T.S., Wu, T.C., Chan, J.W.M., Chu, M.Y., Lee, S.Y., Wong H.Y. and Lung, D.C. (2020).

"Risk of nosocomial transmission of coronavirus disease 2019: an experience in a general ward setting in Hong Kong", Journal of Hospital Infection.

Retrieved on $15^{\text {th }}$ February, 2021 from: : https://www.ncbi.nlm.nih.gov/pubmed/32259546 
World Health Organisation (WHO), (2020). Rolling Updates on Coronavirus Disease (COVID-19). Retrieved on $16^{\text {th }}$ January, 2021 from: https://www.who.int/emergencies/diseases/novel-coronavirus-2019?gclid 